\title{
Persisting symptoms and decreased health-related quality-of-life in a cross-sectional study of treated achalasia patients
}

\author{
R. FRANKHUISEN*, M. A. VAN HERWAARDEN*, R. HEIJKOOP*, A. J. P. M. SMOUT*, A. BARON*, \\ J. R. VERMEIJDEN†, H. G. GOOSZEN* \& M. SAMSOM*
}

*Gastrointestinal Research Unit, Departments of Gastroenterology and Surgery, University Medical Center Utrecht, The Netherlands; †Meander Medical Center, Amersfoort, The Netherlands

Correspondence to:

Dr M. Samsom, Department of Gastroenterology, UMC Utrecht, Heidelberglaan 100, F02.618, 3584 CX Utrecht, The Netherlands.

E-mail: m.samsom@umcutrecht.nl

Publication data

Submitted 28 May 2007

First decision 15 June 2007

Resubmitted 25 June 2007

Accepted 26 June 2007

\section{SUMMARY}

Background

Little is known about symptom characteristics of treated achalasia patients and their effect on health-related quality-of-life (HRQoL).

\section{Aims}

To examine clinical remission, achalasia-associated symptoms and HRQoL in treated achalasia patients.

\section{Methods}

The Eckardt clinical symptom score, RAND-36 and a disease-specific HRQoL questionnaire were sent to 171 treated achalasia patients.

\section{Results}

$76.6 \%$ of the patients returned their questionnaire. $44.9 \%$ of them were not in symptomatic remission. Prevalence of frequent dysphagia (at least daily) and chest pain (at least weekly) was 46\% and 38\%, respectively. Achalasia patients had lower general HRQoL scores than control subjects (all RAND-36 subscales, except health change; $P \leq 0.002$ ). Patients with frequent symptoms of chest pain and dysphagia showed lower HRQoL than patients with less frequent symptoms on three RAND-36 subscales (pain, social functioning and general health perceptions; $P<0.003$ ). Patients in clinical remission showed higher HRQoL than patients who were not, however HRQoL in the 'remission group' remained significantly impaired as compared to controls (all RAND-36 subscales except emotional role limitations and mental health; $P<0.001$ ).

\section{Conclusions}

Many achalasia patients remain severely symptomatic after treatment and have decreased HRQoL. Frequent symptoms are associated with lower HRQoL. Patients in clinical remission show substantially improved, but not restored HRQoL. 


\section{INTRODUCTION}

Achalasia imposes a burden upon patients with this disease in their social life. The disease is clinically characterized by dysphagia and regurgitation of undigested food, caused by aperistalsis of the oesophageal body and the incompetence of the lower oesophageal sphincter (LOS) to relax. ${ }^{1,2}$ Furthermore, chest pain is a common symptom, with a reported prevalence of $60 \%$ among newly diagnosed achalasia patients. ${ }^{3}$ Treatment with (repeated) pneumatic dilation or Heller myotomy can reduce the LOS pressure, thereby relieving symptoms of dysphagia and regurgitation, while chest pain often persists. ${ }^{3}$ Up to now limited and conflicting data have been published regarding the frequency of achalasia-associated oesophageal symptoms in achalasia patients after treatment.

Information concerning health-related quality-of-life (HRQoL) in achalasia patients is limited to studies in which non-validated questionnaires were used and to uncontrolled series after Heller myotomy. ${ }^{4-9}$ Most of these studies report substantially improved HRQoL after Heller myotomy. Data concerning HRQoL after pneumatic dilation is lacking. Besides the limitations of existing data, it is unknown whether remaining achalasia-associated symptoms affect HRQoL or to what extent clinical remission (as defined by symptom scores) is associated with restored HRQoL.

The aims of this study were: 1) to examine the prevalence of achalasia-associated symptoms in a crosssectional group of treated achalasia patients; 2) to investigate the general and disease-specific HRQoL in these patients, as compared to control subjects; and 3) to examine the impact of clinical remission and specific achalasia-associated symptoms on HRQoL.

\section{MATERIAL AND METHODS}

\section{Patients}

A cross-sectional survey was conducted among 171 treated achalasia patients who were treated in our clinic ( $n=136$ patients) and two large affiliated hospitals ( $n=17$ and $n=18)$ between 2000 and 2005. The patients in this study were retrieved via manometric charts or from endoscopic dilation registry and were included if achalasia was diagnosed with oesophageal manometry in accordance with the current criteria. ${ }^{10}$ In all patients upper gastrointestinal endoscopy had been performed to exclude other causes of manometric abnormalities. Patients were approached by mail, nonresponders were called after 6 weeks. Control subjects, without any known chronic medical diseases, were recruited by advertisements in newspapers. These control subjects had neither history of gastrointestinal disease, nor any symptoms that pointed in that direction and no medical history and/or medication use that affected gastrointestinal motility and/or gastric secretion.

In our hospitals it is the standard regime that treated achalasia patients visit our out-patient clinic at least once a year, or more often on demand. If symptoms are severe or increased, oesophageal manometry and timed barium oesophagram are repeated in order to obtain objective parameters. The type of treatment is chosen in consultation with the patient after explaining risks and benefits. Standard treatment consists of three times pneumatic dilation using 3-, 3.5- and 4-cm balloons (Rigiflex; Microvasive, Watertown, MA, USA) at separate sessions ${ }^{11}$ or laparoscopic Heller myotomy with Toupet fundoplication. Patients with insufficient response or quick relapse after pneumatic dilation are offered Heller myotomy. Patients on high surgical risk are treated with Botulinum toxin (25 units injected into each of the four quadrants of the LOS for a total of 100 units).

\section{Questionnaires}

The type and dates of treatment(s) were collected from the case records and consisted of: primary pneumatic dilatation, primary Heller myotomy, Heller myotomy after pneumatic dilation and Botox. All other data were collected from the sent questionnaires. Patients were asked to complete four questionnaires.

(i) The frequency of achalasia-associated symptoms was graded with the use of a previously described clinical symptom score from Eckardt et al. ${ }^{3,12}$ On this scale the frequency of dysphagia and regurgitation (none, occasionally, daily or several times a day) and the degree of weight loss (none, $<5 \mathrm{~kg}, 5-10 \mathrm{~kg}$, $>10 \mathrm{~kg}$ ) were scored on a 0-3 point scale. Chest pain was scored on a 0-4 point scale (none, less than monthly, monthly, weekly, daily). ${ }^{3}$ So, a 0-13 point scale is obtained. Based on previous reports a total score $\leq 4$ was considered as indicative of clinical remission. $^{3}$

(ii) All patients and control subjects completed the RAND-36 questionnaire. This is a validated Dutch translation of the 36-item internationally validated 
general health-related quality of life (HRQoL) questionnaire RAND-36. ${ }^{13-16}$ It contains nine scales: physical functioning, social functioning, physical role limitations, emotional role limitations, mental health, vitality, pain and general health perceptions. RAND-36 item scores are transformed to a 0-100 point scale for each subscale: ${ }^{17}$ a higher score indicates a better HRQoL. The subscales general health perceptions, vitality and mental health will theoretically never reach the maximum score of 100 , because these subscales are bipolar in nature. Only respondents, who evaluate their health favourably, will score a maximum of 100 on these scales.

(iii) A translated and validated version of a diseasespecific HRQoL questionnaire, recently published by Urbach et al., was used. ${ }^{18,19}$ The questionnaire includes 10 achalasia-targeted items addressing dysphagia, dysphagia-related behaviour modifications, pain, heartburn, lifestyle limitation and satisfaction. For each patient a total score was calculated by summing the score for each item (lowest score 10 points, highest score 31 points), where after these outcomes are transformed to a $0-100$ point scale. A lower score represents a better HRQoL.

(iv) A questionnaire inquiring about the onset of achalasia-associated symptoms, the date of diagnosis, use of reflux medication and demographic characteristics.

Control subjects filled out the RAND-36 form.

\section{Analysis}

All data are shown as means and standard deviation (s.d.) unless noted otherwise. For each patient the clinical symptom score, disease specific HRQoL score, use of reflux medication, duration of the disease (defined as the period between the diagnosis and the completion of the questionnaires) and time since last treatment (defined as the period between the last treatment and completion of the questionnaires) were calculated. The RAND-36 outcomes were calculated for each subscale in both achalasia patients and control subjects. We arbitrarily divided patients into those with frequent symptoms, defined as dysphagia $\geq$ once daily, regurgitation $\geq$ once daily and chest pain $\geq$ once a week and those with less frequent symptoms. With this dichotomy, the effect of symptoms on RAND-36 scores and disease specific HRQoL scores were evaluated. Furthermore, the effect of duration of the disease ( $\leq 5$ years vs. $>5$ years), time since last treatment
( $\leq 5$ years vs. $>5$ years) and clinical remission were assessed on the RAND-36 scores and disease specific HRQoL scores. Comparison between groups was performed with a Kruskall-Wallis test and Mann-Whitney $\mathrm{U}$ test. To correct for the number of equations, $P \leq 0.006$ was considered significant.

\section{RESULTS}

\section{Subjects}

One hundred and thirty-one (76.6\%) of 171 patients responded. Five patients were excluded because of insufficient command of the Dutch language or incomplete questionnaires.

Finally, the questionnaires of 126 patients [70 males; median age 48 years (interquartile range (IQR) 40-60 years), 58 females; median age 48 years (IQR 38-65 years); $P=\mathrm{NS}$ ] and 118 controls [58 males; median age 44 years (IQR 31-60 years), 60 females; median age 52 years (IQR 45-58 years); $P=$ NS] were used for analysis. Control subjects fulfilled the same age- and gender distribution as the achalasia patients. Median time since the diagnosis was 9.5 years (IQR 216 years) and median time interval since last treatment 4.6 years (IQR 0.6-16 years). Seventy-two patients (57.1\%) were treated with at least one session of three pneumatic dilations, while seven patients (5.6\%) underwent Heller myotomy as primary treatment. Thirty-nine patients (31.0\%) underwent Heller myotomy as secondary treatment after pneumatic dilation. Eight patients (6.3\%) were treated with Botulinum toxin. Thirty-six percent of the patients used anti-reflux medication at the time of the interview.

\section{Symptom scores}

The median symptom score for all patients was 4 (IQR 2-6). At the moment of their interview 56 patients (44.9\%) were not in clinical remission. Forty-six percent of the patients reported dysphagia $\geq$ once daily, $38.1 \%$ reported chest pain $\geq$ once a week and 25\% complained of both symptoms. Regurgitation $\geq$ once daily was reported by $11.1 \%$ of the patients. Further details on symptom characteristics are summarized in Table S1 (published online as supplementary material). There was no difference in median clinical symptom scores between those patients treated in a tertiary referral centre and the affiliated centres [4 (IQR 2-6) and 4 (IQR 1-5) respectively; $P=$ NS]. 


\section{RAND-36 scores and disease-specific HRQoL scores}

All RAND-36 scores, except health change, were significantly lower in achalasia patients compared with control subjects, with the greatest differences on the RAND-36 subscales physical role limitations, bodily pain and general health perceptions (Figure 1). The total duration of the disease, duration since last treatment and the type of hospital (tertiary referral centre or affiliated hospital) did not affect HRQoL (data not shown).

RAND-36 scores of achalasia patients in clinical remission were significantly higher than those of achalasia patients who were not but still significantly lower compared with control subjects (Figure 2). Improved, but still impaired HRQoL was also found on the disease-specific HRQoL questionnaire when comparing patients in clinical remission to those who were not [39.0 (s.d. 12.4) vs. 55.2 (s.d. 10.7) respectively; $P<0.001$; lower scores represent better HRQoL; see Figure S1 published online as supplementary material].

Patients with frequent symptoms (chest pain $\geq$ once a week and dysphagia $\geq$ once daily) showed lower RAND-36 scores compared with those patients without on the RAND-36 subscales social functioning, pain and general health perceptions (Figure 3). This was also observed on the disease-specific questionnaire: the presence of frequent symptoms caused lower disease-specific HRQoL compared with those achalasia patients without [56.8 (12.2) vs. 37.2 (13.1); $P<0.001$; lower scores represent better HRQoL; see Figure S2 published online as supplementary material].

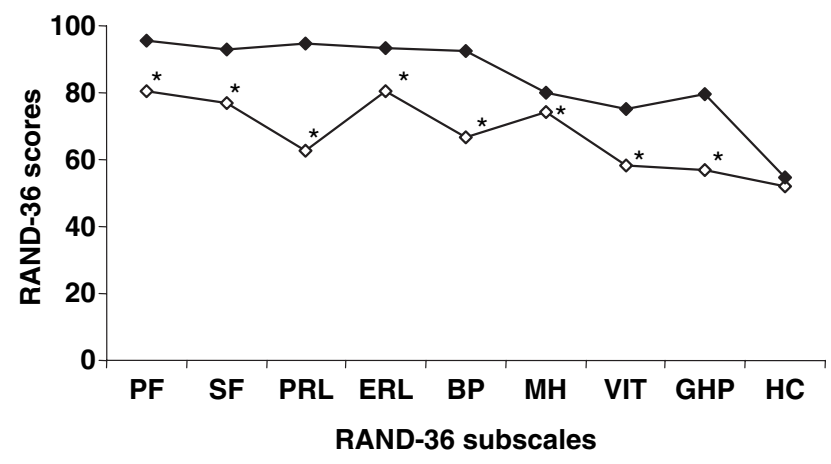

Figure 1. Mean RAND-36 scores are lower in achalasia patients $(\diamond ; n=126)$ than in control subjects ( $\bullet ; n=118)$. ${ }^{*} P<0.002$ vs. control subjects. PF, Physical Functioning; SF, Social Functioning; PRL, Physical Role Limitations; ERL, Emotional Role Limitations; BP, Bodily Pain; MH, Mental Health; VIT, Vitality; GHP, General Health Perceptions; HC, Health Change.

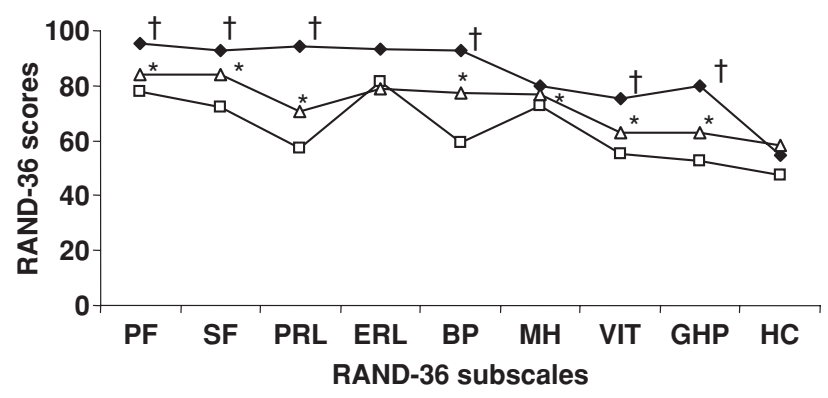

Figure 2. Mean RAND-36 scores in treated achalasia patients in clinical remission $(\triangle ; n=70)$ are higher than in those who are not $(\square ; n=56)$ but do not restore to RAND-36 scores of control subjects ( $\bullet n=118$ ). ${ }^{*} P<0.005$ vs. RAND-36 scores in patients not in clinical remission. $\dagger P<0.001$ vs. RAND-36 scores in patients with or without clinical remission.

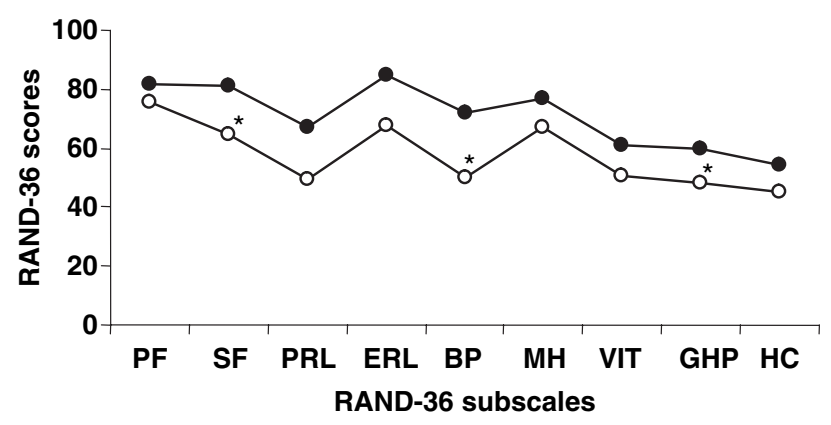

Figure 3. Mean RAND-36 scores in treated achalasia patients with frequent symptoms of chest pain and dysphagia ( $\circ ; n=32$ ) are significantly lower than those without $(\bullet ; n=94)$ on three RAND-36 subscales. ${ }^{*} P<0.003$ vs. RAND-36 scores in patients with less frequent symptoms.

\section{DISCUSSION}

The major findings of this study are that (i) many treated achalasia patients are not in clinical remission (defined as a clinical severity score $\leq 4$ ) due to a high prevalence of dysphagia and chest pain (ii) treated achalasia patients score lower HRQoL compared with a control population and (iii) patients in clinical remission have higher HRQoL than those who are not, but their HRQoL is still lower than that of a symptom-free control population.

This is the first cross-sectional study assessing HRQoL and the presence of persistent symptoms in a large sample of treated achalasia patients in which they were interviewed with validated questionnaires. We showed that only $55.1 \%$ of the treated patients were in clinical remission. This percentage is rather low compared to the 
often mentioned success rates after treatment varying between $60-90 \% .^{20}$ The low remission rate in our study is caused by the high percentage of chest pain (38.1\% reported this symptom at least weekly) and dysphagia (46\% still reported this symptom at least daily). This apparent discrepancy may be caused by differences in questionnaires that were used. For instance, several studies did not evaluate chest pain after treatment while others only assessed the presence and not the frequency of chest pain..$^{92-25}$ As shown by our results, this affects remission rate substantially. In the present study we have chosen to use the symptom score, developed by Eckardt et al. as it has been used in several prospective trials and is focused on the frequency of the three main symptoms of achalasia (dysphagia, regurgitation and chest pain) and also quantifies weight loss. ${ }^{3,12,26}$ The high percentage of chest pain in our study seems in line with percentages reported by Eckardt et al. who found chest pain in 64 out of 101 achalasia patients at initial presentation. In their group only 16\% reported disappearance of this symptom after pneumatic dilation. ${ }^{3}$ The unknown pathophysiological mechanism of chest pain fits within the inability to treat this symptom. A second explanation for the low percentage of clinical remission is the variation in length of follow-up. Zerbib et al. reported a remission rate of $78.8 \%$ with a median follow-up of 3.8 years, while in our study median follow-up was 9.5 years. This is corresponding with the disappointing success rates (40-50\%) in achalasia patients who were evaluated more than 15 years after treatment. ${ }^{27,} 28$

The decreased HRQoL scores observed in our study are substantial especially on the subscales physical role limitations, bodily pain and general health perceptions. The majority of these established HRQoL scores of achalasia patients are comparable with non-institutionalized patients after a stroke or with coronary heart disease. ${ }^{29}$ When the data of control subjects are compared to data from the general Dutch population, as published by Aaronson et al., age and disease in all fields lower the scores in the general population. ${ }^{16}$ It is clear that the symptom-free group's scores are consistent with the absence of gastrointestinal complaints and severe co-morbidity that was required for inclusion. Therefore, the low variation in our control subjects justifies the use of a 1:1 ratio of control subjects to patients, rather than a $3: 1$ or $4: 1$ ratio, which is usually recommended for these case-control studies.

To date literature on HRQoL in achalasia is limited. Only one cross-sectional study has been performed in which HRQoL was measured with a non-validated questionnaire in 41 patients treated with pneumatic dilation and 11 patients after Heller myotomy. ${ }^{4}$ Restrictions on social activities were reported by 15\% of the patients, while $9 \%$ of them were restricted in their physical functioning (housework). The RAND-36 outcomes in our study are substantially lower compared with other studies. These studies assessed HRQoL before and after Heller myotomy and measured post-operative SF-36 scores reaching general population values. ${ }^{6,7,24}$ A possible explanation for the observed differences is that inclusion in a prospective study with the additional attention for a patient could introduce a bias. Secondly, we analysed a larger group of treated achalasia patients, while two of the above-mentioned studies were performed in relatively small samples.

We showed that patients in clinical remission have substantially better HRQoL than patients who are not. This was also observed when comparing individual symptoms, i.e. patients with dysphagia $\geq$ once daily and/or chest pain $\geq$ once a week. So, reduction of symptoms improves HRQoL and in fact re-treatment until clinical remission has achieved, seems justified. However, HRQoL does not completely restore in achalasia patients: on both general and disease-specific HRQoL questionnaires scores from control subjects respectively minimal scores were not reached.

In summary, the prevalence of symptoms in treated achalasia patients is high and significantly impairment in HRQoL is experienced in this group compared to a group of healthy control subjects. Clinical remission is associated with substantial better HRQoL but not a complete restored HRQoL. This emphasizes the importance of detailed symptom evaluation in achalasia patients with a validated clinical symptom score. A HRQoL questionnaire could provide additional value in order to critically evaluate those patients in clinical remission.

\section{Ethics committee approval}

This study was conducted after the medical ethics committee at our hospital approved the protocol in August 2005.

\section{ACKNOWLEDGEMENTS}

The authors like to thank St. Antonius Hospital Nieuwegein and Hospital De Gelderse Vallei Ede Wageningen for their participation in this study. Declaration of personal interests: None. Declaration of funding 
interests: R. Frankhuisen was supported by a grant from ALTANA Pharma.

\section{SUPPLEMENTARY MATERIAL}

The following supplementary material is available for this article:

Table S1. Clinical severity scores of all achalasia patients

Figure S1. Effect of clinical remission on disease-specific HRQoL
Figure S2. Effect of frequent symptoms on diseasespecific HRQoL

This material is available as part of the online article from: http://www.blackwell-synergy.com/doi/abs/10.1111/ j.1365-2036.2007.03423.x (This link will take you to the article abstract).

Please note: Blackwell Publishing are not responsible for the content or functionality of any supplementary materials supplied by the authors. Any queries (other than missing material) should be directed to the corresponding author for the article.

\section{REFERENCES}

1 Vantrappen G, Hellemans J. Treatment of achalasia and related motor disorders. Gastroenterology 1980; 79: 144-54.

2 Spiess AE, Kahrilas PJ. Treating achalasia: from whalebone to laparoscope. JAMA 1998; 280: 638-42.

3 Eckardt VF, Stauf B, Bernhard G. Chest pain in achalasia: patient characteristics and clinical course. Gastroenterology 1999; 116: 1300-4.

4 Meshkinpour H, Haghighat P, Meshkinpour A. Quality of life among patients treated for achalasia. Dig Dis Sci 1996; 41: 352-6.

5 Mineo TC, Pompeo E. Long-term outcome of Heller myotomy in achalasic sigmoid esophagus. J Thorac Cardiovasc Surg 2004; 128: 402-7.

6 Ponce M, Ortiz V, Juan M, Garrigues V, Castellanos C, Ponce J. Gastroesophageal reflux, quality of life, and satisfaction in patients with achalasia treated with open cardiomyotomy and partial fundoplication. Am J Surg 2003; 185: 560-4.

7 Ben Meir A, Urbach DR, Khajanchee YS, Hansen PD, Swanstrom LL. Quality of life before and after laparoscopic Heller myotomy for achalasia. Am J Surg 2001; 181: 471-4.

8 Decker G, Borie F, Bouamrirene D, et al. Gastrointestinal quality of life before and after laparoscopic heller myotomy with partial posterior fundoplication. Ann Surg 2002; 236: 750-8.

9 Raftopoulos Y, Landreneau RJ, Hayetian $\mathrm{F}$, et al. Factors affecting quality of life after minimally invasive Heller myotomy for achalasia. J Gastrointest Surg 2004; 8: 233-9.

10 Spechler SJ, Castell DO. Classification of oesophageal motility abnormalities. Gut 2001; 49: 145-51.
11 Vaezi MF, Richter JE. Diagnosis and management of achalasia. American College of Gastroenterology Practice Parameter Committee. Am J Gastroenterol 1999; 94: 3406-12.

12 Eckardt VF, Aignherr C, Bernhard G. Predictors of outcome in patients with achalasia treated by pneumatic dilation. Gastroenterology 1992; 103: 1732-8.

13 Hunt SM, Mckenna SP. Validating the Sf-36. Brit Med J 1992; 305: 645.

14 Mant JWF, Jenkinson C, Murphy MFG, Clipsham K, Marshall P, Vessey MP. Use of the Short Form-36 to detect the influence of upper gastrointestinal disease on self-reported health status. Qual Life Res 1998; 7: 221-6.

15 Ware JE, Sherbourne CD. The Mos 36item short-form health survey (Sf-36): 1. Conceptual-framework and item selection. Med Care 1992; 30: 473-83.

16 Aaronson NK, Muller M, Cohen PD, et al. Translation, validation, and norming of the Dutch language version of the SF-36 Health Survey in community and chronic disease populations. J Clin Epidemiol 1998; 51: 1055-68.

17 Ware J. SF 36 health survey, manuel and interpretation guide. Boston: Medical Outcomes trust, 1994.

18 Urbach DR, Tomlinson GA, Harnish JL, Martino R, Diamant NE. A measure of disease-specific health-related quality of life for achalasia. Am J Gastroenterol 2005; 100: 1668-76.

19 Frankhuisen R, Heijkoop R, Herwaarden $\mathrm{MA}$, et al. Cross-cultural validation of a disease-specific quality-of-life questionnaire in a large sample of Dutch achalasia patients. submitted for publication 2006.

20 Vaezi MF, Richter JE. Current therapies for achalasia: comparison and effi- cacy. J Clin Gastroenterol 1998; 27: 2135.

21 Vela MF, Richter JE, Wachsberger D, Connor J, Rice TW. Complexities of managing achalasia at a tertiary referral center: use of pneumatic dilatation, Heller myotomy, and botulinum toxin injection. Am J Gastroenterol 2004; 99: 1029-36.

22 Codispoti M, Soon SY, Pugh G, Walker WS. Clinical results of thoracoscopic Heller's myotomy in the treatment of achalasia. Eur $J$ Cardiothorac Surg 2003; 24: 620-4.

23 Csendes A, Braghetto I, Henriquez A, Cortes C. Late results of a prospective randomised study comparing forceful dilatation and oesophagomyotomy in patients with achalasia. Gut 1989; 30: 299-304.

24 Mattioli S, Ruffato A, Di Simone MP, Lugaresi ML, D’Ovidio F. Comparison between subjective and objective assessment of the long-term results after the Heller-Dor operation in patients affected by oesophageal achalasia. Dig Liver Dis 2006; 38: 544-51.

25 Sharp KW, Khaitan L, Scholz S, Holzman MD, Richards WO. 100 consecutive minimally invasive Heller myotomies: lessons learned. Ann Surg 2002; 235: 631-8.

26 Eckardt VF, Gockel I, Bernhard G. Pneumatic dilation for achalasia: late results of a prospective follow up investigation. Gut 2004; 53: 629-33.

27 Karamanolis G, Sgouros S, Karatzias G, et al. Long-term outcome of pneumatic dilation in the treatment of achalasia. Am J Gastroenterol 2005; 100: 270-4.

28 West RL, Hirsch DP, Bartelsman JF, et al. Long term results of pneumatic dilation in achalasia followed for more than 5 years. Am J Gastroenterol 2002; 97: $1346-51$.

29 www.rivm.nl. 